\title{
Inheritance and Linkage Mapping of Eastern Filbert Blight Disease Resistance in 'Uebov' Hazelnut
}

\author{
Gehendra Bhattarai ${ }^{1,2}$, Shawn A. Mehlenbacher ${ }^{1,3,5}$, and David C. Smith ${ }^{1,4}$ \\ Department of Horticulture, Oregon State University, 4017 Agriculture and Life Sciences Building, \\ Corvallis, OR 97331
}

\begin{abstract}
AdDitional INDEX words. Corylus avellana, Anisogramma anomala, microsatellite, simple sequence repeat, linkage map
Abstract. Eastern filbert blight (EFB) is a serious fungal disease of european hazelnut (Corylus avellana) in North America. The causal agent is the pyrenomycete Anisogramma anomala, which is native in the eastern United States where it occasionally produces small cankers on the wild american hazelnut $(C$. americana). However, most commercial cultivars of european hazelnut are susceptible. Infection leads to perennial cankers, girdling of branches, and premature tree death. Cultural practices including scouting, pruning out infected branches, and fungicide applications are recommended to slow disease spread but are expensive and not completely effective. EFB resistance from 'Gasaway' is conferred by a dominant allele at a single locus and has been extensively used in the Oregon State University hazelnut breeding program, but there is concern that this resistance could be overcome by isolates now present in the eastern United States or that a new race of the pathogen could arise in Oregon. Segregation for EFB resistance from 'Uebov', a new source from Serbia, was studied in three progenies by a combination of structure exposure and greenhouse inoculation. The frequency of resistant seedlings following structure exposure was about $\mathbf{2 0 \%}$ in all three progenies. The ratios failed to fit the expected 1:1 ratio but did fit a ratio of 1 resistant:3 susceptible, which would be expected if resistance were conferred by dominant alleles at two independent loci. Seedlings from a cross of susceptible selection OSU $\mathbf{7 4 1 . 1 0 5}$ and 'Uebov' were used to study correlation of disease response and presence of alleles at microsatellite marker loci. Resistance was highly correlated with the presence of alleles at marker loci on linkage group 6 (LG6), and these markers also showed segregation distortion. We conclude that EFB resistance from 'Uebov' maps to a single locus on LG6 in the same region as resistance from 'Gasaway', although only about $20 \%$ of the seedlings are resistant because of segregation distortion. 'Uebov' has large, well-filled, round nuts and is suitable as a parent in breeding for the in-shell market, but its low nut yields and a high frequency of shells with split sutures are the drawbacks. Its use would expand options for breeding and 'Uebov' resistance could be combined with other resistance alleles with an expectation of more durable EFB resistance. Durable resistance would not only sustain the hazelnut industry in Oregon but would also allow expansion of plantings to new areas.
\end{abstract}

Hazelnut, also known as filbert, is one of the world's major nut crops. Hazelnut is an important commercial crop in the Willamette Valley of Oregon and acreage is increasing with the planting of cultivars released by Oregon State University. Eastern filbert blight, incited by the pyrenomycete Anisogramma anomala, is a serious threat to Oregon's hazelnut industry. The fungus is native to the eastern United States where it occasionally produces small cankers on the wild american hazelnut. The fungus is an obligate biotroph with a 2-year life cycle that includes a 15 -month latent period between inoculation and symptom expression (Johnson et al., 1996; Pinkerton et al., 1995). This latent period delays disease response phenotyping and slows the breeding process. Stromata are present on the sunken, perennial cankers. When branches become wet in winter and spring, the perithecia swell and eject ascospores because of an increase in pressure (Pinkerton et al.,

Received for publication 26 Apr. 2017. Accepted for publication 26 May 2017. This research partially fulfilled the requirements for a Master of Science degree for G. Bhattarai. Funding was provided by the Oregon Hazelnut Commission, Oregon Agricultural Experiment Station, Hatch Act, a specific cooperative agreement with USDA, USDA-NIFA Agriculture and Food Research Initiative Competitive Grant 2014-67013-22421, and USDA-NIFA Specialty Crops Research Initiative Competitive Grant 2009-51181-06028.

${ }^{1}$ Current address: Program in Cell and Molecular Biology and Department of Horticulture, University of Arkansas, Fayetteville, AR 72701.

${ }^{2}$ Former Graduate Research Assistant.

${ }^{3}$ Professor.

${ }^{4} \mathrm{Sr}$. Faculty Research Assistant.

${ }^{5}$ Corresponding author. E-mail: shawn.mehlenbacher@oregonstate.edu.
1998). The released ascospores are disseminated by wind and rain, and may land on and infect young shoots after budbreak in the spring (Pscheidt, 2010). The spores germinate, and the hyphae penetrate young shoot tips and then colonize the cambium and phloem, leading to the formation of sunken cankers (Johnson et al., 1994; Stone et al., 1992). Fifteen months after infection, the elliptical stromata emerge in the cankers in a single or double row along the axis of the stem. EFB cankers expand annually, leading to the girdling of branches and causing tree death in 6-12 years in the absence of control measures (Pinkerton et al., 1993). Recommended control measures include regular scouting for the presence of the disease in the orchard, pruning infected branches $1 \mathrm{~m}$ below the cankers, and fungicide applications. Four fungicide applications beginning at budbreak (early to mid-March) and repeated at 2-week intervals are recommended (Johnson et al., 1994; Pscheidt and Cluskey, 2001). Although fungicide sprays and therapeutic pruning slow the spread of EFB, these control measures are expensive, reduce nut yield, and are not completely effective (Johnson et al., 1996). Given the environmental and economic concerns associated with chemical control, host genetic resistance is viewed as the most desirable and economical means of controlling the disease.

A dominant allele at a single locus from the obsolete pollinizer 'Gasaway' confers a very high level of resistance (Mehlenbacher et al., 1991), and several recent releases from the Oregon State University hazelnut breeding program carry this resistance. Recent hazelnut releases including 'Jefferson', 
'Yamhill', 'Dorris', 'Wepster', and 'McDonald', the pollinizers 'Eta', 'Theta', 'York', and, 'Felix', and the ornamentals 'Red Dragon' and 'Burgundy Lace' (Mehlenbacher et al., 2009, 2011, 2012, 2013, 2014, 2016) all carry resistance from 'Gasaway'. Random amplified polymorphic DNA (RAPD) markers linked to resistance have been identified (Mehlenbacher et al., 2004), of which UBC152-800 and UBC268-580 are routinely used for marker-assisted selection. The locus was placed on LG6 by Mehlenbacher et al. (2006), and microsatellite markers have been added to the map (Bhattarai 2015; Bhattarai and Mehlenbacher, 2017; Colburn et al., 2017; Gürcan and Mehlenbacher, 2010; Gürcan et al., 2010; Sathuvalli and Mehlenbacher, 2013). Microsatellite markers, also known as simple sequence repeat (SSR) markers, serve as anchors on the linkage map as they map to the same location in multiple progenies. Correlation of disease phenotype with SSR allele scores allows assignment of a resistance locus to a linkage group.

The breeding of resistant cultivars carrying major resistance (R) genes is an effective and economical strategy to control disease. Nevertheless, qualitative resistance genes are not often durable. Large-scale and long-term cultivation of a single cultivar increases selection pressure, and a high mutation rate in the pathogen favors the evolution of new races. Greenhouse inoculation at Rutgers University showed that 'Gasaway' and some of its offspring were infected by one or a few A. anomala isolates (Molnar et al., 2010). Under high disease pressure in Oregon, small cankers have been observed on 'Jefferson' and 'McDonald', which carry resistance from 'Gasaway'. Because of concerns about the durability of 'Gasaway' resistance, the search for new EFB resistance sources continues. In addition to 'Gasaway', EFB resistance has been identified in several $C$. avellana accessions, where it is under the control of dominant alleles. Resistance from four additional sources [OSU 408.040, 'Culplà', 'Crvenje', and OSU 495.072] from Minnesota, Spain, Serbia, and southern Russia, respectively, maps to the same region on LG6 as 'Gasaway' resistance (Colburn et al., 2015; Sathuvalli et al., 2012). Resistance from 'Ratoli', C. americana 'Rush' and interspecific hybrid 'Yoder \#5' map to a region on LG7 (Bhattarai et al., 2017; Sathuvalli et al., 2011a), and from selection OSU 759.010 from the Republic of Georgia to LG2 (Sathuvalli et al., 2011b).

In Oregon and New Jersey, tests have identified EFBresistant selections among the plants grown from seeds collected in Turkey, the Republic of Georgia, Russia, Crimea, Moldova, Poland, and Estonia (Capik et al., 2013; Leadbetter et al., 2015; Molnar et al., 2007; S.A. Mehlenbacher and T.J. Molnar, unpublished data). EFB resistance has also been reported in other Corylus species including the shrubs $C$. americana, C. heterophylla, C. kweichowensis (synonym $C$. heterophylla var. sutchuensis), C. cornuta, and C. californica, and the single-trunk tree hazels C. colurna, C. chinensis, and $C$. fargesii (Capik and Molnar, 2012; Chen et al., 2007; Coyne et al., 1998) as well as several interspecific hybrids.

'Uebov' was received as scions from the ARI Fruit and Grape Research Center in Čačak, Serbia. 'Uebov' has incompatibility alleles S12 and S16 (Mehlenbacher, 2014). 'Uebov' remained free of EFB after inoculation in the greenhouse (Sathuvalli et al., 2010) and field exposure (Capik and Molnar, 2012). When 'Uebov' DNA was amplified with the polymerase chain reaction (PCR) using RAPD primers UBC152 and UBC268, the bands used for marker-assisted selection for 'Gasaway' resistance were absent. In a diversity study of EFB-resistant accessions using microsatellite markers, 'Uebov' was placed in the Spanish-Italian group (Muehlbauer et al., 2014). This article describes the performance of 'Uebov' hazelnut, investigates the inheritance of EFB resistance from 'Uebov', and places the resistance locus on the linkage map.

\section{Materials and Methods}

Nut, KeRnel, AND tree eValuation. 'Uebov' was propagated by grafting scions to rooted layers of $C$. avellana and a single tree was planted in the guard row of a replicated yield trial established in Spring 2003 at the Smith Horticultural Research Farm in Corvallis, OR. Four self-rooted trees each of seven numbered selections and standards 'Barcelona', 'Clark', and 'Lewis' were planted in the trial. Nuts were harvested by hand annually for 5 years (2005-09), dried, and weighed. For 4 years (2006-09), two samples of 10 nuts each from each tree were evaluated for nut weight, kernel weight, and kernel percentage. Annually, in the same 4 years, two samples of 50 nuts from each tree were rated for the amount of fiber on the pellicle, ease of pellicle removal (blanching), and frequency of defects. Ratings of bud mite susceptibility (primarily Phytoptus avellanae) were recorded annually in December on a scale of 1 (no blasted buds) to 5 (many blasted buds). Trunk diameters were measured $30 \mathrm{~cm}$ above the soil line at the end of the 2009 season and used to estimate the trunk cross-sectional area. Yield efficiency was calculated for each tree as the ratio of total nut yield to trunk cross-sectional area.

SEEDLing POPUlations. Three seedling populations were studied (Table 1; Fig. 1). In 2006, 'Uebov' was crossed as the male parent with susceptible selections OSU 675.028 and OSU 679.114 , generating progenies 06030 and 06031 , respectively. In 2007, 'Uebov' was crossed as the male parent with susceptible selection OSU 741.105, generating progeny 07024. Seeds from these controlled pollinations were harvested in August and removed from the husks. In early November, the whole nuts were soaked in water for $2 \mathrm{~d}$ and then stratified at $4{ }^{\circ} \mathrm{C}$ for $3-5$ months. Seeds were planted as root tips emerged, and the resulting seedlings were transplanted to $2.8-\mathrm{L}$ pots and grown in the greenhouse for the summer and either planted in the field in October or placed under the inoculation structure in late winter.

Structure inoculation. Seedlings in pots were moved under the EFB inoculation structure in late winter before leaf budbreak. They were inoculated by exposure for 8 weeks (from late March to early June) as described by Pinkerton et al. (1993). Diseased branches with mature EFB cankers were collected at the Oregon State University Smith Horticulture Research Farm in early January and stored under polyethylene in an unheated building for use as inoculum. In Spring 2008, 112 seedlings of progeny 06030 and 115 seedlings of progeny 06031 were exposed as potted trees under the structure. In Spring 2009, 147 seedlings of progeny 07024 were structureinoculated. Potted seedlings were placed on the ground where a wooden frame supported a wire mesh suspended above the plants. Diseased branches were placed on the mesh in late March, and additional infected branches were added 4 weeks later. Irrigation sprinklers installed above the mesh and inoculum were turned on daily beginning at leaf budbreak for a total of $60 \mathrm{~min}$ each morning to ensure branch wetness and spore discharge. After the seedling trees were exposed, they 
Table 1. Nut yields, trunk cross-sectional area (TCA), yield efficiency, and bud mite ratings of three hazelnut cultivars and selection OSU 741.105 in a replicated trial planted in 2003. A single grafted tree of 'Uebov' was planted in the guard row.

\begin{tabular}{|c|c|c|c|c|c|c|c|c|c|c|}
\hline \multirow[b]{2}{*}{ Cultivar } & \multirow[b]{2}{*}{ Trees (no.) } & \multicolumn{6}{|c|}{ Nut yield (kg/tree) } & \multirow[b]{2}{*}{ TCA $\left(\mathrm{cm}^{2}\right)^{\mathrm{z}}$} & \multirow{2}{*}{$\begin{array}{c}\text { Yield efficiency } \\
\left(\mathrm{kg} \cdot \mathrm{cm}^{-2}\right)^{\mathrm{z}}\end{array}$} & \multirow{2}{*}{$\begin{array}{l}\text { Bud mite rating } \\
(1 \text { to } 5 \text { scale })^{\mathrm{y}}\end{array}$} \\
\hline & & 2005 & 2006 & 2007 & 2008 & 2009 & Total & & & \\
\hline OSU 741.105 & 4 & 0.25 & 2.21 & 4.66 & 3.13 & 11.18 & 21.42 & 102.2 & 0.21 & 1.0 \\
\hline Barcelona & 2 & 0.18 & 2.75 & 3.59 & 2.96 & 8.68 & 18.16 & 159.1 & 0.11 & 1.0 \\
\hline Lewis & 4 & 0.51 & 4.02 & 3.35 & 7.14 & 9.38 & 24.40 & 88.5 & 0.28 & 2.5 \\
\hline Uebov & 1 & 0.05 & 1.04 & 2.25 & 2.48 & 3.82 & 9.64 & 65.5 & 0.15 & 1.0 \\
\hline $\operatorname{LSD}(0.05)$ & & 0.25 & 0.71 & 1.06 & 1.32 & 2.21 & 3.42 & 14.9 & 0.04 & 0.3 \\
\hline
\end{tabular}

$\overline{{ }^{\mathrm{z}} \mathrm{TCA}}=$ trunk cross-sectional area calculated from trunk diameter $30 \mathrm{~cm}$ above the soil line in Dec. 2009, Yield efficiency $=$ total nut yield/TCA.

${ }^{\mathrm{y}}$ Susceptibility to bud mite (primarily Phytoptus avellanae) rated annually for 4 years on a scale of 1 (no blasted buds) to 5 (many blasted buds).
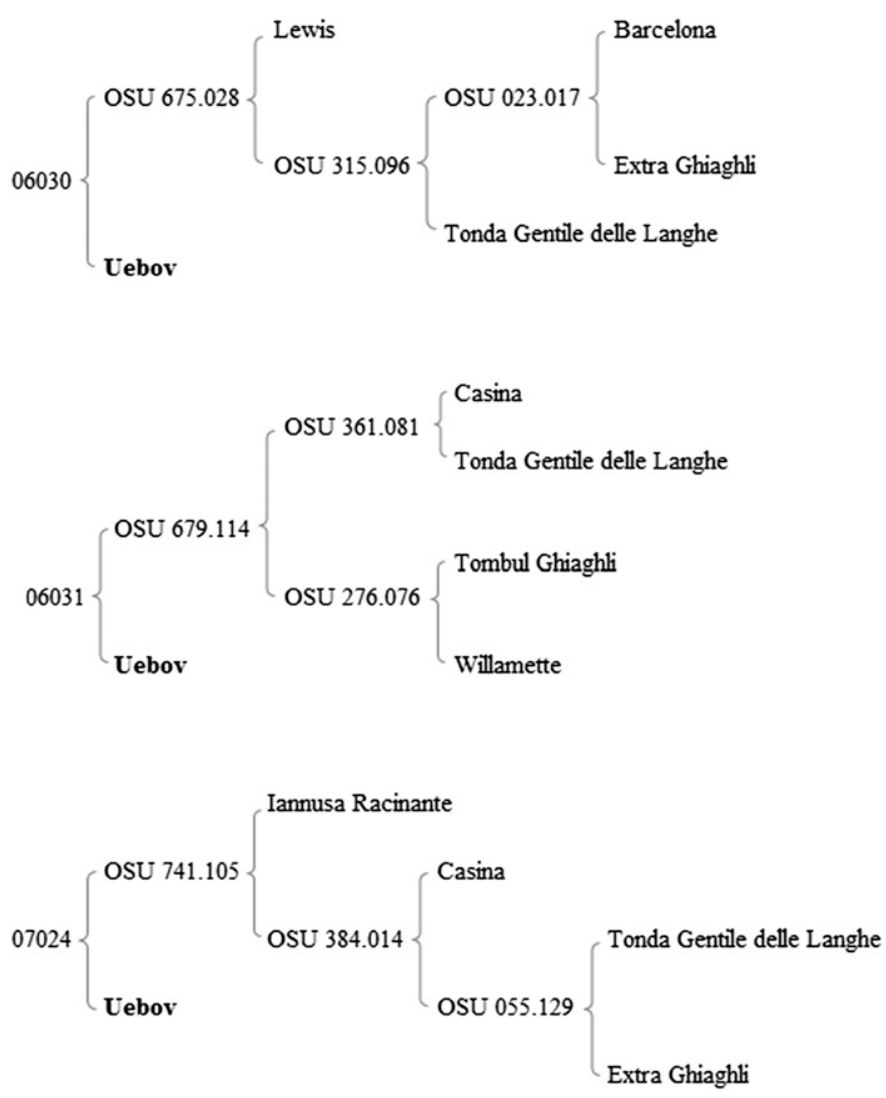

Fig. 1. Pedigrees of hazelnut progenies 06030,06031 , and 07024 segregating for eastern filbert blight resistance from 'Uebov'. Only 'Uebov' is resistant; all other selections are susceptible.

were transplanted to nursery rows where they were grown and disease severity was scored $\approx 20$ months after inoculation. In early January, seedlings were observed for the presence of cankers and stromata, and disease severity was scored on a scale of 0 to 5 , with 0 being the absence of disease symptoms and 5 being very severe disease symptoms. Seedlings with a very small canker $(<10 \mathrm{~cm})$ but no stromata were given a rating of 1 . Seedlings with a very small canker and a few stromata were given a rating of 2 . Seedlings with stromata were given ratings of 3,4 , or 5 with a larger amount of dead wood leading to a higher score. For analysis, seedlings with ratings of 0,1 , or 2 were considered resistant and those with ratings of 3,4 , or 5 were considered susceptible. Three potted self-rooted trees of 'Ennis' (highly susceptible), four of 'Daviana' (highly susceptible) and four of 'Tonda di Giffoni' (high quantitative resistance) were placed under the inoculation structure with the seedlings as controls in the 2008 exposure. Three trees of 'Ennis', five of 'Tonda di Giffoni' and eight of 'Dorris' (very high resistance from 'Gasaway') served as checks in the 2009 exposure.

Greenhouse inOCulations. On 26 Oct. 2007, 115 seedlings of progeny 06030 were planted in a field plot, and on 8 Oct. 2008, 125 seedlings of progeny 07024 were planted in a different field plot. These are different seedlings from two of the progenies used in structure inoculations. In Dec. 2012, scions were collected in the field from seedlings of these two progenies and stored at $-1{ }^{\circ} \mathrm{C}$ until grafting. Three scions of each selection were grafted to rooted layers of $C$. avellana in Apr. 2013 and potted in 6-L pots containing equal volumes of fine bark of douglas fir (Pseudotsuga meziesii) bark, pumice, and peat. The trees were grown in the glasshouse with $24{ }^{\circ} \mathrm{C}$ days and $18{ }^{\circ} \mathrm{C}$ nights until the plants grew vigorously and had developed four to five nodes. Diseased wood with cankers bearing mature stromata was collected at the Oregon State University Smith Horticulture Research Farm in Corvallis in Dec. 2012 and stored in polyethylene bags at $-20{ }^{\circ} \mathrm{C}$ until late spring and early summer when they were used for greenhouse inoculations. EFB inoculum was prepared $<3 \mathrm{~h}$ before inoculation. Stored diseased wood was removed from the freezer, thawed at room temperature, placed in a plastic box, and covered with a moist paper towel. Perithecia were extracted from the stromata using a scalpel, hydrated in a small amount of distilled water for a few minutes, and then ground with mortar and pestle to release ascospores. The suspension was filtered through cheesecloth, water was added to bring the spore suspension to $100 \mathrm{~mL}$, and ascospore concentration was determined using a hemacytometer (Hausser Scientific, Horsham, $\mathrm{PA})$. The suspension was diluted to a concentration of $1 \times 10^{6}$ spores $/ \mathrm{mL}$. In the preparation for inoculation, grafted trees with actively growing shoot tips were chosen, the shoot tips were marked with colored paper tape two to three nodes below the apical meristem to indicate the point of inoculation, and the trees placed in inoculation chambers. Tape of a different color was used on each of the four inoculation dates in 2013 (green, blue, white, and pink on 21 June, 8 July, 22 July, and 30 July, respectively) to facilitate record-keeping. The inoculation chambers were described by Colburn et al. (2015). The shoot tips were inoculated by spraying the spore suspension on the shoot tips until wet using a spray bottle. Two inoculations were performed, in either early morning (before $0700 \mathrm{HR}$ ) or late evening (after $2000 \mathrm{HR}$ ), and the second inoculation was performed $3 \mathrm{~d}$ after the first inoculation. Three misters per 
bench spaced $0.3 \mathrm{~m}$ apart with a flow of $7.75 \mathrm{~L} \cdot \mathrm{h}^{-1}$ were mounted $0.9 \mathrm{~m}$ above each benchtop. The misters were programmed to run for $10 \mathrm{~s}$ every $30 \mathrm{~min}$ during the day (0800-1900 HR) and $10 \mathrm{~s}$ every hour during the night (1900$0800 \mathrm{HR}$ ). The inoculations were conducted during the cool hours of the day to increase the success rate and minimize the number of escapes (false negatives). Three days following the second inoculation, the trees were removed from the inoculation chamber, placed on benches, and grown for 3-6 months under the greenhouse conditions described previously. 'Ennis' (14 potted layers), 'Tonda di Giffoni' (16 potted layers), and the parents OSU 675.028 (five grafted trees) and OSU 741.105 (nine grafted trees) were included as susceptible controls, with 'Uebov' (11 grafted trees) as the resistant control. In late summer, the potted trees were moved to a shady area at the Smith Horticultural Research Farm, allowed to harden, and then transplanted to a nursery row in mid-October. Disease responses were scored in Dec. 2014, 16-18 months after inoculation. Disease severity was rated on individual trees using the same 0 to 5 scale as for structure inoculated seedlings. A genotype was scored as resistant if all three inoculated trees remained free of cankers and stromata, or susceptible if one or more trees developed cankers and stromata. When shoot tips showed dieback before the emergence of stromata, a note of "top dead" was recorded for that tree.

DNA EXTRACTION, AMPLIFICATION WITH MICROSATELLITE PRIMER PAIRS, AND MAPPING. DNA was extracted from the parents and seedlings of progeny 07024 (OSU 741.105 and 'Uebov'). From plants growing at the Oregon State University Smith Horticultural Research Farm, two to four very young leaves were collected in Spring 2013 and DNA extracted, quantified, and diluted with TE buffer to a concentration of $20 \mathrm{ng} \cdot \mu \mathrm{L}^{-1}$ as described by Colburn et al. (2015). DNA from the two parents was amplified using previously mapped SSR markers, starting with markers on LG6 near the 'Gasaway' resistance locus and continuing, as needed, to markers on other linkage groups. PCR mixtures $(10 \mu \mathrm{L})$ contained $0.3 \mu \mathrm{M}$ each of the fluorescent forward and the nonfluorescent reverse primer, $1 \times \mathrm{NH}_{4}$ reaction buffer (Biolase; Bioline USA, Taunton, MA), $2 \mathrm{~mm} \mathrm{MgCl}_{2}, 200 \mu \mathrm{M}$ each of dATP, dCTP, dGTP, and dTTP, $20 \mathrm{ng}$ template DNA, and 0.25 units of DNA polymerase (Biolase; Bioline USA). PCR amplifications in 96-well plates were performed in thermal cyclers (GeneAmp PCR system 9700; Applied Biosystems, Foster City, CA). The program was as follows: denaturation at $95^{\circ} \mathrm{C}$ for $5 \mathrm{~min}$ followed by 40 cycles of $94^{\circ} \mathrm{C}$ for $40 \mathrm{~s}, 60^{\circ} \mathrm{C}$ for $40 \mathrm{~s}, 72^{\circ} \mathrm{C}$ for $40 \mathrm{~s}$, extension at $72{ }^{\circ} \mathrm{C}$ for $7 \mathrm{~min}$, and a final infinite hold at $4{ }^{\circ} \mathrm{C}$. The presence of an amplification product was inspected by separating 3-4 $\mu \mathrm{L}$ of PCR product by electrophoresis on $2.5 \%$ agarose gels at $100 \mathrm{~V}$ for $1 \mathrm{~h}$, staining with ethidium bromide, destaining in water, and then visualizing under ultraviolet light using a BioDoc-It ${ }^{\circledR}$ Imaging System (UVP, Upland, CA). For allele sizing, amplified products were multiplexed such that different fluorescent dyes were used, and there was no overlap in size. Two microliters each of 4-10 SSR products were diluted in distilled water to a final volume of $150 \mu \mathrm{L}$. An aliquot $(1-1.5 \mu \mathrm{L})$ of the multiplex was submitted to the Core Laboratories of Oregon State University's Center for Genome Research and Biocomputing (CGRB) for fragment sizing by capillary electrophoresis on an ABI 3730 machine (Life Technologies, Carlsbad, CA). Allele sizes were determined using GeneMapper ${ }^{\circledR}$ software (Life Technologies) and fragment lengths were entered in a spreadsheet. PCR amplification and capillary electrophoresis were repeated when no fragment was amplified or the result was ambiguous. When the parental SSR marker fingerprints indicated that segregation would occur in the seedlings, DNA of 23 resistant and 23 susceptible seedlings and the parents was amplified. For the initial correlation analysis, disease response and marker scores for the 46 seedlings were entered in columns in a spreadsheet. Resistant seedlings were scored as 1, and susceptible seedlings were scored as 0 . Likewise, SSR alleles were scored as present (1) or absent (0). Pearson product-moment correlation coefficients were then calculated for disease response and SSR allele scores. The LG was considered assigned when the absolute value of the correlation coefficient $(|r|)$ was greater than 0.5 . When the LG had been identified, DNA of 138 seedlings in progeny 07024 was amplified using primer pairs of several additional SSRs on the same LG. The seedlings included 109 previously untested seedlings that were inoculated in the greenhouse, and 29 additional seedlings that had been scored as resistant following an earlier structure inoculation (Table 3). Each allele was scored as present or absent, and the marker data were used to construct a genetic map.

DAta Analysis. Data for nut yield per tree, trunk crosssectional area, yield efficiency, and bud mite scores were subjected to an analysis of variance using the GLM procedure of SAS (version 9.4; SAS Institute, Cary, NC). Data for nut weight, kernel weight, kernel percentage, ratings for fiber and pellicle removal, and the frequency of good kernels and seven types of defects were also analyzed with SAS. After structure exposure, a seedling was considered resistant if given a score of 0,1 , or 2 , and susceptible if given a rating of 3,4 , or 5 . After greenhouse inoculation, a selection was considered resistant if all three grafted trees remained free of disease; if one or more trees showed a sunken canker with stromata, the selection was considered susceptible. Chi square tests were used to determine goodness-of-fit to expected segregation ratios. Heterogeneity chi square tests were performed to determine if the data from the progenies could be pooled.

MAPPING. After initial assignment of resistance to a $\mathrm{LG}$, DNA of a total of 138 seedlings of progeny 07024 was amplified with primer pairs of several polymorphic SSR loci from that LG. At loci segregating in ratios of $1: 1$ and $1: 1: 1: 1$, alleles were scored in each seedling as 1,0 , or $n$ for presence, absence, and unknown status. Similarly, markers segregating in a 1:2:1 ratio were scored as 1 for homozygous presence of the allele, 0 for homozygous absence of the allele, $n$ for heterozygous condition, and $n$ for unknown status. The marker scores 1 , 0 , and $n$ were then replaced with JoinMap's codes $h, a$, and $u$, respectively, and the file saved in tab-delimited text format. The file was imported into JoinMap software (version 4.1; Kyazma, Wageningen, The Netherlands) and mapping conducted using the $\mathrm{BC} 1$ population type. The linkage map was constructed using the maximum likelihood (ML) mapping algorithm with default recombination frequency of 0.40 , a logarithm of the odds $(\mathrm{LOD})=10$ threshold for grouping and map distances shown in Haldane units (centimorgans). Markers present in repulsion phase were included by creating "dummy variables" in which " $h$ " was replaced with " $a$," and " $a$ " was replaced with " $h$." The use of dummy variables allowed merger of repulsion phase markers with coupling phase markers to generate a single map for each parent. The map output was inspected for "Fit and Stress" values and markers that fit poorly 
Table 2. Nut weight, kernel weight, kernel percentage, ratings for fiber and pellicle removal, and frequency of nut and kernel defects for three hazelnut cultivars and selection OSU 741.105 in a replicated trial planted in 2003. A single grafted tree of 'Uebov' was planted in the guard row.

\begin{tabular}{|c|c|c|c|c|c|c|c|c|c|c|c|c|c|c|}
\hline \multirow[b]{2}{*}{ Cultivar } & \multirow[b]{2}{*}{$\begin{array}{l}\text { Trees } \\
\text { (no.) }\end{array}$} & \multirow[b]{2}{*}{$\begin{array}{c}\text { Nut } \\
\text { wt }(g)^{z}\end{array}$} & \multirow[b]{2}{*}{$\begin{array}{l}\text { Kernel } \\
\text { wt }(g)^{\mathrm{z}}\end{array}$} & \multirow[b]{2}{*}{$\begin{array}{c}\text { Kernel } \\
\text { percentage }^{\mathrm{y}}\end{array}$} & \multirow[b]{2}{*}{$\begin{array}{c}\text { Fiber } \\
(1-4 \text { scale })^{\mathrm{x}}\end{array}$} & \multirow{2}{*}{$\begin{array}{c}\text { Pellicle } \\
\text { removal } \\
(1-7 \text { scale })^{\mathrm{w}}\end{array}$} & \multicolumn{8}{|c|}{ Frequency $(\%)^{\mathrm{v}}$} \\
\hline & & & & & & & Good & Blank & $\begin{array}{c}\text { Brown } \\
\text { stain }\end{array}$ & $\begin{array}{l}\text { Poor } \\
\text { fill }\end{array}$ & Shriveled & Moldy & $\begin{array}{c}\text { Black } \\
\text { tips }\end{array}$ & Twin \\
\hline OSU 741.105 & 4 & 2.39 & 1.18 & 49.4 & 3.0 & 2.8 & 85.3 & 4.7 & 0.0 & 3.5 & 0.9 & 3.8 & 1.0 & $\overline{0.9}$ \\
\hline Clark & 4 & 2.49 & 1.29 & 51.6 & 2.5 & 3.4 & 80.4 & 1.9 & 1.4 & 10.1 & 1.4 & 4.1 & 0.5 & 0.6 \\
\hline Lewis & 4 & 2.91 & 1.37 & 47.0 & 1.1 & 4.2 & 71.4 & 3.1 & 0.1 & 14.7 & 2.5 & 7.8 & 0.3 & 0.7 \\
\hline Uebov & 1 & 4.31 & 2.02 & 46.9 & 2.0 & 3.3 & 71.8 & 2.0 & 0.0 & 13.8 & 0.5 & 5.0 & 5.5 & 2.0 \\
\hline
\end{tabular}

${ }^{\mathrm{z}}$ Means for nut weight and kernel weight are based on samples of 20 nuts per tree for 4 years (2006-09).

${ }^{\mathrm{y}}$ Kernel percentage $=$ kernel weight/nut weight.

${ }^{\mathrm{x}}$ Fiber on pellicle rated from 1 (none) to 4 (much).

${ }^{\mathrm{w}}$ Pellicle removal (blanching) rated on a scale of 1 (complete pellicle removal) to 7 (no pellicle removal) after roasting at $150{ }^{\circ} \mathrm{C}$ for $15 \mathrm{~min}$ and rubbing.

${ }^{v}$ Mean frequencies of good nuts and defects for 4 years (2006-09). Samples of 100 nuts were evaluated from each tree in each year.

Table 3. Disease responses following inoculation of hazelnut seedlings with eastern filbert blight in structure and greenhouse tests and goodnessof-fit to $1: 1$ and 1:3 ratios. 'Uebov' is resistant, and the three numbered selections are susceptible.

\begin{tabular}{|c|c|c|c|c|c|c|c|c|c|}
\hline \multirow[b]{2}{*}{$\mathrm{Yr}^{\mathrm{z}}$} & \multirow[b]{2}{*}{ Progeny } & \multirow[b]{2}{*}{ Parentage } & \multicolumn{3}{|c|}{ Disease response } & \multirow{2}{*}{$\begin{array}{l}\text { Expected } \\
\text { ratio }\end{array}$} & \multirow[b]{2}{*}{$\chi^{2}$} & \multirow[b]{2}{*}{ df } & \multirow[b]{2}{*}{$\mathrm{P}$} \\
\hline & & & Resistant & Susceptible & Unknown $^{y}$ & & & & \\
\hline \multicolumn{10}{|l|}{ Structure inoculation } \\
\hline \multirow[t]{2}{*}{2008} & 06030 & OSU $675.028 \times$ Uebov & 24 & 88 & - & $1: 1$ & 36.57 & 1 & 0.00 \\
\hline & & & & & & $1: 3$ & 0.76 & 1 & 0.38 \\
\hline \multirow[t]{2}{*}{2008} & 06031 & OSU $679.114 \times$ Uebov & 23 & 92 & - & $1: 1$ & 41.40 & 1 & 0.00 \\
\hline & & & & & & $1: 3$ & 1.53 & 1 & 0.22 \\
\hline \multicolumn{10}{|l|}{ Greenhouse inoculation } \\
\hline \multirow[t]{2}{*}{2013} & 06030 & OSU $675.028 \times$ Uebov & 20 & 68 & 15 & $1: 1$ & 19.36 & 1 & 0.00 \\
\hline & & & & & & $1: 3$ & 0.48 & 1 & 0.49 \\
\hline \multirow[t]{3}{*}{2013} & 07024 & OSU $741.105 \times$ Uebov & 28 & 72 & 9 & $1: 1$ & 18.67 & 1 & 0.00 \\
\hline & & & & & & $1: 3$ & 0.57 & 1 & 0.45 \\
\hline & & & $24^{\mathrm{x}}$ & $72^{\mathrm{x}}$ & 13 & $1: 1$ & 24.00 & 1 & 0.00 \\
\hline Heterogeneity & & & & & & & 2.38 & 4 & 0.67 \\
\hline
\end{tabular}

${ }^{\mathrm{z}}$ Year of disease inoculation.

${ }^{y}$ Disease response unknown because shoot tips of all grafted trees died before stromata emerged.

"After inspecting disease and marker scores, four seedlings initially called resistant (DC14, DD45, DD61, and DB50) were reclassified as "Unknown." The original scores were used for the heterogeneity chi square calculation.

(high values) were removed in stepwise manner. Linkage maps were drawn with MapChart software (Kyazma).

\section{Results}

Nut, KERNEL, AND TREE EVAlUATION. Although 'Uebov' was represented by a single grafted tree, comparisons with standard cultivars in the trial are useful (Tables 1 and 2). Four trees of 'Barcelona' were planted, but one died shortly after planting and a second tree had a damaged trunk so its size and productivity were atypical. Data are presented for the two healthy 'Barcelona' trees and four trees each of selection OSU 741.105 and the standards 'Lewis' and 'Clark'. 'Uebov' is highly resistant to bud mites and its total nut yield was less than all of the control cultivars. The tree size was small and similar to 'Clark'. Nut yield efficiency is low and similar to
'Barcelona'. The nuts are round, attractive, and borne in clusters of one or two in husks $25 \%$ longer than the nuts. The nuts fall free of the husk at maturity, which is a few days later than 'Barcelona'. Nuts and kernels are a bit larger than 'Barcelona' and thus suited to the in-shell market. There is little fiber on the pellicle, and most of the pellicle is removed by the blanching process; thus, raw and blanched kernels are attractive. The nuts are $47 \%$ kernel by weight, and the shells have weak sutures. As a result of the weak sutures, the two halves of the shell sometimes split at the suture, leading to kernels with black tips and the entrance of kernel mold.

Structure test of EFB Resistance in 'Uebov' PROGEnies. Seedlings of three progenies were exposed to EFB under a structure topped with diseased branches and the percentage of resistant seedlings was similar $(20.0 \%$ to $21.4 \%)$. This percentage is far below the $50 \%$ expected if 'Uebov' were 
Table 4. Disease responses following greenhouse inoculation of trees of parent and standard hazelnut genotypes with eastern filbert blight.

\begin{tabular}{llccc}
\hline & \multicolumn{2}{c}{$\begin{array}{c}\text { Disease response in } \\
\text { pultivar or selection }\end{array}$} & \multicolumn{2}{c}{ Disease response in this test (no. trees) } \\
\cline { 2 - 5 } & Highly susceptible & 0 & 2 & Unknown $^{z}$ \\
\hline Ennis & Quantitative resistance & $2^{\mathrm{y}}$ & 1 & 10 \\
Tonda di Giffoni & Highly resistant & 6 & 0 & 5 \\
Uebov & Susceptible & 0 & 2 & 3 \\
OSU 675.028 & Susceptible & 0 & 3 & 6 \\
OSU 741.105 & & 0 &
\end{tabular}

${ }^{\mathrm{z}}$ Disease response unknown because shoot tips of all trees died before stromata emerged.

${ }^{\mathrm{y}}$ Two trees of 'Tonda di Giffoni' had small sunken cankers that lacked stromata.

Table 5. Segregation for eastern filbert blight resistance in hazelnut progeny 07024 (OSU $741.105 \times$ 'Uebov') and at linked microsatellite marker loci on linkage group 6. Goodness-of-fit tests are for overall segregation ratios and transmission of alleles from each parent [female (F), male $(M)$ ]. Shown in bold is the allele linked to resistance, and in the right column, the correlation of the marker and disease resistance scores.

\begin{tabular}{|c|c|c|c|c|c|c|c|c|}
\hline Locus & Alleles $(+\times \widehat{0})$ & Expected ratio & Observed ratio & Missing $^{\mathrm{z}}$ & $\chi^{2}$ & $\mathrm{df}$ & $P$ & $|r|^{\mathrm{y}}$ \\
\hline$\overline{\mathrm{GB} 602}$ & $233 / 239 \times 233 / 239$ & $1: 2: 1$ & $41: 77: 19$ & 1 & 9.18 & 2 & 0.01 & 0.69 \\
\hline \multirow[t]{3}{*}{ A614 } & $124 / 131 \times 126 / 141$ & 1:1:1:1 & $37: 15: 55: 16$ & 15 & 35.54 & 3 & 0.00 & - \\
\hline & & $1: 1 \mathrm{~F}(124)$ & $52: 71$ & 15 & 2.93 & 1 & 0.09 & - \\
\hline & & $1: 1 \mathrm{M}(141)$ & $31: 92$ & 15 & 30.25 & 1 & 0.00 & 0.69 \\
\hline \multirow[t]{3}{*}{ LG688 } & $359 / 362 \times 362 / 368$ & $1: 1: 1: 1$ & $25: 37: 24: 51$ & 1 & 13.98 & 3 & 0.00 & - \\
\hline & & $1: 1 F(359)$ & $62: 75$ & 1 & 1.23 & 1 & 0.27 & - \\
\hline & & $1: 1 \mathrm{M}(368)$ & $49: 88$ & 1 & 11.10 & 1 & 0.00 & 0.87 \\
\hline Uebov & $\mathrm{rr} \times \mathbf{R r}$ & $1: 1 \mathrm{M}$ & $54: 71$ & 13 & 2.31 & 1 & 0.13 & - \\
\hline \multirow[t]{3}{*}{ LG668 } & $0.165 \times 0 / \mathbf{1 7 2}^{\mathrm{y}}$ & $1: 1: 1: 1$ & $28: 48: 25: 36$ & 1 & 9.25 & 3 & 0.03 & - \\
\hline & & $1: 1 \mathrm{~F}(16$ & $61: 76$ & 1 & 1.64 & 1 & 0.20 & - \\
\hline & & $1: 1 \mathrm{M}(17$ & $53: 84$ & 1 & 7.01 & 1 & 0.01 & 0.92 \\
\hline \multirow[t]{3}{*}{ LG682 } & $393 / 426 \times 412 / 430$ & $1: 1: 1: 1$ & $40: 27: 31: 26$ & 14 & 3.94 & 3 & 0.27 & 一 \\
\hline & & $1: 1 F(393)$ & $67: 57$ & 14 & 0.81 & 1 & 0.37 & - \\
\hline & & $1: 1 \mathrm{M}(430)$ & $53: 71$ & 14 & 2.61 & 1 & 0.11 & 0.90 \\
\hline \multirow[t]{3}{*}{ LG631 } & $429 / 434 \times \mathbf{4 2 8} / 434$ & $1: 1: 1: 1$ & $29: 43: 27: 37$ & 2 & 4.82 & 3 & 0.19 & 一 \\
\hline & & $1: 1 \mathrm{~F}(42$ & $72: 64$ & 2 & 0.47 & 1 & 0.49 & - \\
\hline & & $1: 1 \mathrm{M}(428)$ & $56: 80$ & 2 & 4.24 & 1 & 0.04 & 0.90 \\
\hline LG639 & $228 / 228 \times \mathbf{2 3 2} / 235$ & $1: 1 \mathrm{M}(232)$ & $54: 77$ & 7 & 4.04 & 1 & 0.04 & 0.90 \\
\hline LG628 & $217 / 217 \times \mathbf{2 1 5} / 217$ & $1: 1 \mathrm{M}(215)$ & $58: 78$ & 2 & 2.94 & 1 & 0.09 & 0.84 \\
\hline
\end{tabular}

${ }^{\mathrm{z}}$ Microsatellite marker not scored for this number of seedlings.

${ }^{\mathrm{y}}$ At locus LG668, alleles 159 and 162 were present in all seedlings, whereas segregation was observed and scored for alleles 165 and 172.

heterozygous at a single locus and resistance were dominant. However, the progenies showed good fit to a 1:3 ratio and indicate the possible control of disease response by two independent loci. The heterogeneity chi square, calculated using an expected ratio of 1:3 (Table 3), showed that data for the three progenies could be pooled with each other and with data from the greenhouse inoculations.

Greenhouse test of EFB resistance in 'Uebov' PROGENIES. The greenhouse test of grafted trees was complicated by a high frequency of plants marked "top dead" (118 of 367 in progeny 06030 and 108 of 402 in progeny 07024 ), indicating that the inoculated shoots died before when stromata would have emerged and provide no information on their EFB susceptibility. A selection was considered resistant only when none of the grafted had stromata that produced spores (Table 3 ). When all three trees were rated "top dead," that seedling's disease phenotype was scored as "unknown" for segregation analysis and map construction. Nine seedlings of progeny 07024 and 15 of progeny 06030 fell into this category. In progeny 06030,20 seedlings were scored as resistant and 68 as susceptible, while disease response could not be determined for the remaining 15 . In progeny 07024,28 seedlings were scored as resistant and 72 as susceptible, with the remaining nine seedlings having all trees marked "top dead." There was a deficiency of resistant seedlings in both progenies and neither fit a 1:1 ratio, but both fit a ratio of 1 resistant: 3 susceptible. The heterogeneity chi square test, calculated using an expected ratio of 1:3 (Table 3), showed that data for the two greenhouse-inoculated progenies could be pooled with each other and with the data from structure inoculation. Some trees of control cultivars as well as seedlings were marked "top dead." Of the parents and checks (Table 4), 'Uebov' was resistant, whereas OSU 675.028, OSU 741.105 , and the highly susceptible 'Ennis' were scored as susceptible. In 'Tonda di Giffoni', a control with a high level of quantitative resistance, two trees developed small sunken cankers that lacked stromata, and one developed normal cankers. A total of 37 trees of the parents and checks were rated as "top dead."

SSR MARKER ANALYSIS AND LINKAGE MAPPING. The initial analysis using 46 seedlings in progeny 07024 showed a high correlation of resistance and presence of allele 430 at marker locus LG682 $(r=-0.72)$. This locus had been previously mapped to LG6 near the 'Gasaway' resistance locus (Sathuvalli and Mehlenbacher, 2013), so nearby marker loci were investigated. Parental scores indicated that two loci (LG639 and LG628) were expected to segregate in a 1:1 ratio, five loci (LG631, LG688, A614, LG668, and LG682) were expected to segregate in a 1:1:1:1 ratio, and GB602 was expected to segregate in a 1:2:1 ratio. At three additional SSR markers previously assigned to LG6, GB671 and GB310 did not segregate in progeny 07024 , and LG648 was heterozygous only in the susceptible female parent and so information from this progeny was not useful for mapping the resistant parent 'Uebov'. Eight SSR marker loci previously mapped to the same region on LG6 were heterozygous in 'Uebov' and were used to amplify DNA of 138 trees (57 resistant, 72 susceptible, and 9 unknown disease response). Correlation coefficients for disease response and marker scores in the 138 seedlings at the 8 loci ranged from 0.69 to 0.92 (Table 5). JoinMap placed the scores for eight markers and disease response in a single group at a LOD score of 13. Values for "nearest neighbor stress (cM)" and "nearest neighbor fit (cM)" were low, indicating good fit with the adjacent markers. After the initial mapping, marker 


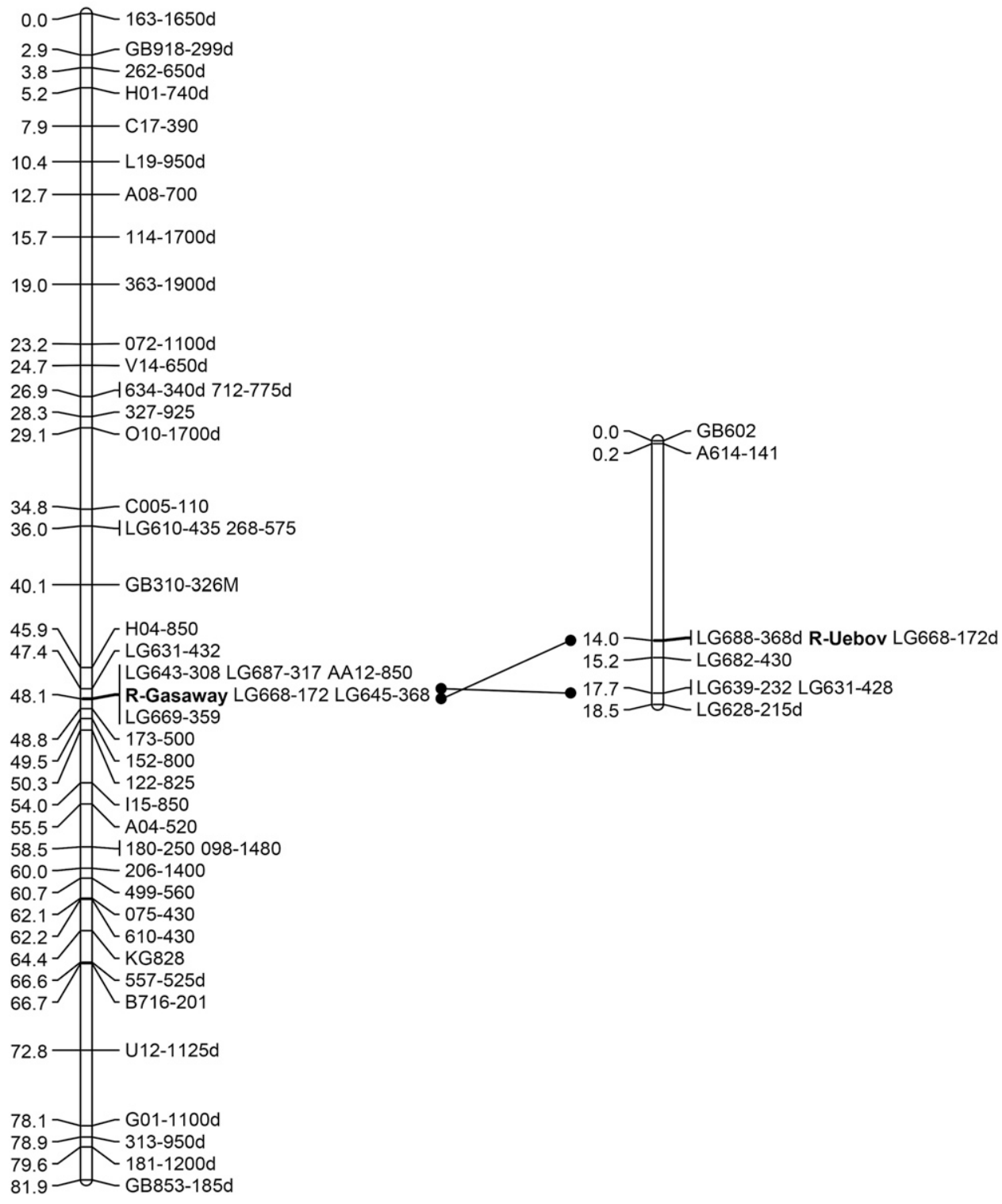

Fig. 2. Aligned maps of Linkage Group 6 for hazelnut progeny 07024 (right) segregating for eastern filbert blight resistance from 'Uebov' $(n=138)$ and the reference map for hazelnut population OSU $252.146 \times$ OSU 414.062 (left). The eastern filbert blight resistance loci are shown in bold. Microsatellite markers developed by Bhattarai (2015) are shown with a GB prefix. In constructing the map for progeny 07024, the scores of four selections that escaped infection were changed from resistant to unknown.

scores for the 138 seedlings were aligned in a spreadsheet and recombinants and suspicious disease phenotypes identified. Three seedlings (DC14, DD45, and DD61) had been scored as resistant based on a single tree of each, and one seedling (DB50) had been scored as resistant based on two trees. The other grafted trees of these seedlings had been scored as "top dead." It appears that these four seedlings are susceptible but escaped infection. Their disease response was then changed to "unknown," giving a new ratio of 24 resistant:72 susceptible:13 unknown, plus 29 additional resistant seedlings from structure exposure. A new map was constructed with scores from these 138 seedlings (Fig. 2). The genetic linkage map of the resistant parent 'Uebov' (Fig. 2) spans $18.5 \mathrm{cM}$ and shows EFB resistance cosegregating with microsatellite markers
LG668 and LG688, with LG682 only $1.2 \mathrm{cM}$ away. Although segregation ratios failed to fit a $1: 1$ ratio, the mapping indicates control of disease response by a single locus with distorted segregation, and similar distortion was noted at the nearby microsatellite marker loci (Table 5). LG628 and LG639 were expected to segregate $1: 1$, yet both show a deficiency of the allele linked to resistance. Loci A614, LG688, LG668, LG682, and LG631 were expected to segregate $1: 1: 1: 1$, which allowed separate goodness-of-fit tests for alleles in the two parents. In all five progenies, segregation in the female parent showed good fit to expectation (1:1), but segregation in the resistant parent 'Uebov' showed a deficiency of the allele linked to resistance (Table 5).

\section{Discussion}

Seedlings with an EFB rating of 0 had no disease symptoms and were considered resistant, while those with ratings of 3,4 , or 5 had fully developed stromata and were clearly susceptible. Trees with ratings of 1 or 2 are more problematic, but very few trees were given these ratings. Following structure inoculation, only five seedlings in progeny 06030 and three in progeny 07024 were given a score of 1 or 2. Many grafted trees inoculated in the greenhouse and transplanted to the nursery row had dead shoot tips, and this would have prevented the emergence of stromata. It appears that these trees had suffered cold injury during two cold weather events, the first in Dec. 2013 and the second in Feb. 2014. Such cold events are rare in Corvallis. Selections segregating for resistance from other sources, including 'Gasaway', were also classified as "top dead" in this set of inoculations (data not shown). The "top dead" classification was much more frequent in this test than in prior tests and does not appear to be related to 'Uebov' parentage. If resistance is governed by a dominant allele at a single locus, one expects transmission of resistance to $50 \%$ of the offspring. However, in all three progenies, there was an excess of susceptible seedlings $(\approx 80 \%)$ and all failed to fit a $1: 1$ ratio. However, they did fit a 1:3 ratio and ratios for five populations (three in structure inoculation, two in greenhouse inoculation) were homogeneous $\left(\chi^{2}=2.10, \mathrm{df}=4, P=0.72\right)$ and were pooled. This suggested the involvement of two independent loci in resistance, with the resistant parent being heterozygous at both loci and susceptible parents recessive at both 
loci. An alternative explanation is that a single locus is involved in 'Uebov' resistance but with distorted segregation. Indeed, distorted segregation for disease response was observed in all three progenies (Table 3), as well as at SSR markers linked to resistance (Table 5), which supports the explanation of control by a single locus with distorted segregation. In spite of the low transmission of resistance, the nuts of 'Uebov' are large and round, and the kernels fill the shells. This is a rare combination among hazelnut cultivars, making 'Uebov' a desirable parent for the in-shell market where nuts of this type are prized.

Deviations from an expected 1:1 segregation ratio have been reported in previous EFB resistance studies in hazelnut. Of the 19 progenies segregating for EFB resistance from 'Zimmerman' hazelnut, only 2 fit an expected ratio of 1:1 whereas the remaining 17 fit a ratio of 3 resistant: 1 susceptible (Lunde et al., 2006). Sathuvalli et al. (2011b) studied segregation in two progenies for EFB resistance from Georgian selection OSU 759.010. One progeny segregated in a ratio of $1: 1$ while the other showed a ratio of 3 resistant: 1 susceptible, although resistance mapped to a single locus on LG2. Similarly, resistance from 'Crvenje', 'Culplà', and Russian selection OSU 495.072 mapped to LG6 but transmission of EFB resistance varied widely among the progenies (Colburn et al., 2015). Two progenies of 'Crvenje' showed an excess of susceptible seedlings. Of two progenies of 'Culplà', one fit a 1:1 ratio while the other showed better fit to a ratio of 3 resistant: 1 susceptible. Of the five progenies segregating for resistance from OSU 495.072, three fit a ratio of 1:1, one fit a ratio 3 resistant: 1 susceptible, and one had an excess of resistant seedlings but did not fit either a $1: 1$ or a $3: 1$ ratio. Likewise, Bhattarai et al. (2017) studied five progenies segregating for EFB resistance from selection 'Yoder \#5' and found that three showed good fit to a $1: 1$ ratio, one fit this ratio at $P=0.05$, and one had an excess of resistant seedlings. Chromosomal abnormalities are a possible cause of such irregular segregations. Reciprocal translocations are common in hazelnut cultivars. Salesses and Bonnet (1988) observed quadrivalents or trivalents at meiosis in 'Barcelona' and 'Tonda Gentile delle Langhe', and both of these are present in the pedigree of 06030 and 'Tonda Gentile delle Langhe' is present in the pedigrees of 06031 and 07024.

To date, EFB resistance loci have been mapped to three linkage groups $(6,2$, and 7). Resistance from 'Gasaway', OSU 408.040, 'Crvenje', 'Culplà', Russian selection OSU 495.072 and now 'Uebov' map to LG6 (Colburn et al., 2015; Mehlenbacher et al., 2006; Sathuvalli et al., 2012), from 'Ratoli', C. americana 'Rush' and interspecific hybrid 'Yoder \#5' to LG7 (Bhattarai et al., 2017; Sathuvalli et al., 2011a) and from Georgian OSU 759.010 to LG2 (Sathuvalli et al., 2011b). Linked markers identified in these studies will be useful for pyramiding these resistance genes. Moreover, markers tightly linked to the resistance locus are important for map-based cloning, sequencing, and chromosome walking. Leadbetter et al. (2016) investigated transmission of resistance in crosses of resistant Russian and Crimean selections with susceptible selections. Six selections transmitted resistance to about $50 \%$ of their offspring, while five others transmitted resistance to about $25 \%$ of their offspring. Additional sources of EFB resistance have been identified, including those of Molnar et al. (2007), but inheritance has not yet been studied. The planting of cultivars with one major resistance gene over a large area often causes directional selection leading to an increase in the frequency of virulent races and a decrease in the frequency of avirulent pathotypes. The eventual stacking of these different resistant sources is a rational approach for breeding cultivars with broad-spectrum EFB resistance that will be more durable, as several mutations would be needed to overcome the multiple $\mathrm{R}$ genes.

The assignment of several resistance sources to LG6 and LG7 prompts the question of whether they are the same resistance gene or different loci in a cluster. R-gene clusters have been reported in many plant species, including common bean [Phaseolus vulgaris (David et al., 2009)], coffee [Coffea arabica (Ribas et al., 2011)], and apple [Malus $\times$ domestica (Xu and Korban, 2002)]. Fine mapping, cloning, and functional characterization of candidate genes will eventually elucidate the disease resistance mechanism (Schouten et al., 2014). Whole-genome resequencing and the alignment of the sequences that include the resistance locus and flanking markers would be useful in identifying candidate genes. RNA sequencing and differential expression studies would complement these studies to identify functional genes associated with disease resistance. Additionally, tightly linked markers could be identified or developed for each different resistance source, eventually making the pyramiding of disease resistance genes precise and practical in hazelnut. For resistance genes located on different chromosomes, the stacking of $\mathrm{R}$ genes is straightforward. In a clonal crop, such as hazelnut, it is also possible to stack resistance alleles at the same locus or nearby loci in a cluster in a single heterozygous clonally propagated cultivar. The pyramiding of two or more dominant resistance genes in a single genotype is an approach that is expected to enhance disease resistance durability, particularly if the genes are unique and have different resistance mechanisms.

\section{Literature Cited}

Bhattarai, G. 2015. Microsatellite marker development, characterization and mapping in european hazelnut (Corylus avellana L.), and investigation of novel sources of eastern filbert blight resistance in several Corylus species. MS Thesis, Oregon State Univ., Corvallis, OR.

Bhattarai, G. and S.A. Mehlenbacher. 2017. In silico development and characterization of tri-repeat microsatellite markers in hazelnut (Corylus avellana L.). PLoS One 12(5):e0178061.

Bhattarai, G., S.A. Mehlenbacher, and D.C. Smith. 2017. Eastern filbert disease resistance from Corylus americana 'Rush' and selection 'Yoder \#5' maps to linkage group 7. Tree Genet. Genomes 13(2):45.

Capik, J.M. and T.J. Molnar. 2012. Assessment of host (Corylus sp.) resistance to eastern filbert blight in New Jersey. J. Amer. Soc. Hort. Sci. 137:157-172.

Capik, J.M., M. Muehlbauer, A. Novy, J.A. Honig, and T.J. Molnar. 2013. Eastern filbert blight resistant hazelnuts from Russia, Ukraine, and Poland. HortScience 48:466-473.

Chen, H., S.A. Mehlenbacher, and D.C. Smith. 2007. Hazelnut accessions provide new sources of resistance to eastern filbert blight. HortScience 42:466-469.

Colburn, B.C., S.A. Mehlenbacher, and V.R. Sathuvalli. 2017. Development and mapping of microsatellite markers from transcriptome sequences of european hazelnut (Corylus avellana L.) and use for germplasm characterization. Mol. Breed. 37(2):16 , doi: 10.1007/ s11032-016-0616-2.

Colburn, B.C., S.A. Mehlenbacher, V.R. Sathuvalli, and D.C. Smith. 2015. Eastern filbert blight resistance in hazelnut accessions 'Culplà', 'Crvenje' and OSU 495.072. J. Amer. Soc. Hort. Sci. 140: 191-200. 
Coyne, C.J., S.A. Mehlenbacher, and D.C. Smith. 1998. Sources of resistance to eastern filbert blight in hazelnut. J. Amer. Soc. Hort. Sci. 123:253-257.

David, P., N.W. Chen, A. Pedrosa-Harand, V. Thareau, M. Sévignac, S.B. Cannon, D. Debouck, T. Langin, and V. Geffroy. 2009. A nomadic subtelomeric disease resistance gene cluster in common bean. Plant Physiol. 151:1048-1065.

Gürcan, K. and S.A. Mehlenbacher. 2010. Development of microsatellite marker loci for european hazelnut (Corylus avellana L.) from ISSR fragments. Mol. Breed. 26:551-559.

Gürcan, K., S.A. Mehlenbacher, R. Botta, and P. Boccacci. 2010. Development, characterization, segregation, and mapping of microsatellite markers for european hazelnut (Corylus avellana L.) from enriched genomic libraries and usefulness in genetic diversity studies. Tree Genet. Genomes 6:513-531.

Johnson, K.B., S.A. Mehlenbacher, J.K. Stone, J.W. Pscheidt, and J.N. Pinkerton. 1996. Eastern filbert blight of hazelnut: It's becoming a manageable disease. Plant Dis. 80:1308-1316.

Johnson, K.B., J.N. Pinkerton, S.M. Gaudreault, and J.K. Stone. 1994. Infection of european hazelnut by Anisogramma anomala: Site of infection and effect of host developmental stage. Phytopathology 84:1465-1470.

Leadbetter, C.W., J.M. Capik, S.A. Mehlenbacher, and T.J. Molnar. 2016. Hazelnut accessions from Russia and Crimea transmit resistance to eastern filbert blight. J. Amer. Pomol. Soc. 70:92-109.

Leadbetter, C.W., J.M. Capik, M. Pisetta, and T.J. Molnar. 2015. Sources of resistance to eastern filbert blight in hazelnuts from the Republic of Georgia. Sci. Hort. 193:269-275.

Lunde, C.F., S.A. Mehlenbacher, and D.C. Smith. 2006. Segregation for resistance to eastern filbert blight in progeny of 'Zimmerman' hazelnut. J. Amer. Soc. Hort. Sci. 131:731-737.

Mehlenbacher, S.A. 2014. Geographic distribution of incompatibility alleles in cultivars and selections of European hazelnut. J. Amer. Soc. Hort. Sci. 139:191-212.

Mehlenbacher, S.A., R.N. Brown, J.W. Davis, H. Chen, N.V. Bassil, D.C Smith, and T.L. Kubisiak. 2004. RAPD markers linked to eastern filbert blight resistance in Corylus avellana. Theor. Appl. Genet. 108:651-656.

Mehlenbacher, S.A., R.N. Brown, E.R. Nouhra, T. Gokirmak, N.V. Bassil, and T.L. Kubisiak. 2006. A genetic linkage map for hazelnut (Corylus avellana L.) based on RAPD and SSR markers. Genome 49:122-133.

Mehlenbacher, S.A., D.C. Smith, and R. McCluskey. 2009. 'Yamhill' hazelnut. HortScience 44:845-847.

Mehlenbacher, S.A., D.C. Smith, and R.L. McCluskey. 2011. 'Jefferson' hazelnut. HortScience 46:662-664.

Mehlenbacher, S.A., D.C. Smith, and R.L. McCluskey. 2012. 'Eta' and 'Theta' hazelnut pollenizers. HortScience 47:1180-1181.

Mehlenbacher, S.A., D.C. Smith, and R.L. McCluskey. 2013. 'Dorris' hazelnut. HortScience 48:796-799.

Mehlenbacher, S.A., D.C. Smith, and R.L. McCluskey. 2014. 'Wepster' hazelnut. HortScience 49:346-349.

Mehlenbacher, S.A., D.C. Smith, and R.L. McCluskey. 2016. 'McDonald' hazelnut. HortScience 51:757-760.

Mehlenbacher, S.A., M.M. Thompson, and H.R. Cameron. 1991. Occurrence and inheritance of resistance to eastern filbert blight in 'Gasaway' hazelnut. HortScience 26:410-411.

Molnar, T.J., J.C. Goffreda, and C.R. Funk. 2010. Survey of Corylus resistance to Anisograma anomala from different geographic locations. HortScience 45:832-836.
Molnar, T.J., D.E. Zaurov, J.C. Goffreda, and S.A. Mehlenbacher. 2007. Survey of hazelnut germplasm from Russia and Crimea for response to eastern filbert blight. HortScience 42:51-56.

Muehlbauer, M.F., J.A. Honig, J.M. Capik, J.N. Vaiciunas, and T.J. Molnar. 2014. Characterization of eastern filbert blight-resistant hazelnut germplasm using microsatellite markers. J. Amer. Soc. Hort. Sci. 139:399-432.

Pinkerton, J.N., K.B. Johnson, S.A. Mehlenbacher, and J.W. Pscheidt. 1993. Susceptibility of european hazelnut clones to eastern filbert blight. Plant Dis. 77:261-266.

Pinkerton, J.N., K.B. Johnson, J.K. Stone, and K.L. Ivors. 1998. Factors affecting the release of ascospores of Anisogramma anomala. Phytopathology 88:122-128.

Pinkerton, J.N., J.K. Stone, S.J. Nelson, and K.B. Johnson. 1995. Infection of european hazelnut by Anisogramma anomala: Ascospore adhesion, mode of penetration of immature shoots, and host response. Phytopathology 85:1260-1268.

Pscheidt, J.W. 2010. Eastern filbert blight help page. 1 Dec. 2012. $<\mathrm{http}$ ://oregonstate.edu/dept/botany/epp/EFB/>.

Pscheidt, J.W. and S. Cluskey. 2001. Fungicide control of eastern filbert blight. Acta Hort. 556:411-418.

Ribas, A.F., A. Cenci, M.C. Combes, H. Etienne, and P. Lashermes. 2011. Organization and molecular evolution of a disease-resistance gene cluster in coffee trees. BMC Genomics 12(1):240.

Salesses, G. and A. Bonnet. 1988. Etude cytogénétique d'hybrides entre variétés de noisetier (Corylus avellana) porteuses d'une translocation à l'état heterozygote. Cytologia (Tokyo) 53:407-413.

Sathuvalli, V.R., H. Chen, S.A. Mehlenbacher, and D.C. Smith. 2011a. DNA markers linked to eastern filbert blight resistance in 'Ratoli' hazelnut (Corylus avellana L.). Tree Genet. Genomes 7:337-345.

Sathuvalli, V.R. and S.A. Mehlenbacher. 2013. De novo sequencing of hazelnut bacterial artificial chromosomes (BACs) using multiplex Illumina sequencing and targeted marker development for eastern filbert blight resistance. Tree Genet. Genomes 9:1109-1118.

Sathuvalli, V.R., S.A. Mehlenbacher, and D.C. Smith. 2010. Response of hazelnut accessions to greenhouse inoculation with Anisogramma anomala. HortScience 45:1116-1119.

Sathuvalli, V.R., S.A. Mehlenbacher, and D.C. Smith. 2011b. DNA markers linked to eastern filbert blight resistance from a hazelnut selection from the Republic of Georgia. J. Amer. Soc. Hort. Sci. 136:350-357.

Sathuvalli, V.R., S.A. Mehlenbacher, and D.C. Smith. 2012. Identification and mapping of DNA markers linked to eastern filbert blight resistance from OSU 408.040 hazelnut. HortScience 47:570 573

Schouten, H.J., J. Brinkhuis, A. van der Burgh, J.G. Schaart, R. Groenwold, G.A. Broggini, and C. Gessler. 2014. Cloning and functional characterization of the Rvi15 (Vr2) gene for apple scab resistance. Tree Genet. Genomes 10:251-260.

Stone, J.K., K.B. Johnson, J.N. Pinkerton, and J.W. Pscheidt. 1992. Natural infection period and susceptibility of vegetative seedlings of european hazelnut to Anisograma anomala. Plant Dis. 76:348352.

Xu, M. and S.S. Korban. 2002. A cluster of four receptor-like genes resides in the $V f$ locus that confers resistance to apple scab disease. Genetics 162:1995-2006. 